\title{
QSAR-ANALYSIS OF POLYSUBSTITUTED FUNCTIONALIZED AMINOTHIAZOLES WITH ANTIHYPERTENSIVE ACTIVITY
}

\author{
I.V. Drapak \\ DANYLO HALYTSKY LVIV NATIONAL MEDICAL UNIVERSITY, LVIV, UKRAINE
}

Background. QSAR analysis is an important tool for the identification of pharmacophore fragments in biologically active substances and helps optimize the search for new effective drugs.

Objective. The aim of the study was to determine the molecular descriptors for QSAR analysis of polysubstituted functionalized aminothiazoles as a theoretical basis for purposeful search de novo of potential antihypertensive drugs among the investigated compounds.

Methods. Calculation of molecular descriptors and QSAR-models creation was carried out using the HyperChem 7.5 and BuildQSAR packages.

Results. The calculation of a number of molecular descriptors (electronic, steric, geometric, energy) was performed for 15 new polysubstituted functionalized aminothiazoles, with established in vivo antihypertensive activity. According to the calculated molecular descriptors and antihypertensive activity parameter, the QSAR models were derived $H A=a+b \cdot X 1+c \cdot X 2+d \cdot X 3$, where the activity parameter $H A$ is antihypertensive activity and $X 1, X 2, X 3$ are molecular descriptors.

Conclusion. The study of 'the structure - antihypertensive activity' relationship for polysubstituted functionalized aminothiazoles was carried out. QSAR analysis revealed that volume, area, lipophilicity, dipole moment, refractivity, polarization of the molecule and energy of the lowest unoccupied molecular orbital have the most significant effect on antihypertensive activity. It was suggested that the attained QSAR-models may have antihypertensive activity within abovementioned row of compounds and can be considered as theoretical basis for de novo design of new potential antihypertensive drugs.

KEY WORDS: polysubstituted functionalized aminothiazoles; antihypertensive activity; molecular descriptors; QSAR-analysis.

\section{Introduction}

QSAR analysis is an important tool for identification of pharmacophore fragments in biologically active substances and helps optimize the search for new effective drugs. To date, systematic correlation analysis is a necessary step for lead-compounds identification in the search for a new drug. The directed search for new biologically active substances can be succeeded by the use of virtual methods of research, which are carried out by means of QSAR/QSRR analysis (quantitative structureactivity/quantitative structure-property relationship), which allows establishing quantitative patterns of relation between the activity or properties of the investigated compounds and the parameters of their molecular structure. Quantitative Structure - Activity Relationship is nowadays widely used as a method for predicting the biological activity of new compounds. [1-3].

Corresponding Author: Iryna Drapak, PhD, Department of General, Bioinorganic, Physical and Colloidal Chemistry, Danylo Halytsky Lviv National Medical University, 69 Pekarska, 79010, Lviv, Ukraine.Email - iradrapak@ukr.net
Thiazole derivatives are a promising class for the search for biologically active compounds, since the thiazole nucleus is a strong biophore fragment for the rational design of 'drug-like molecules', and thiazole derivatives exhibit various types of biological activity: anti-inflammatory [4], antihypertensive [5], cardioprotective [6], antioxidant [7].

The objectieve of the study was to determine the molecular descriptors for QSAR analysis of the new polysubstituted functionalized aminothiazoles as a theoretical basis for search for new antihypertensive drugs among abovementioned types of compounds.

\section{Methods}

15 polysubstituted functionalized aminothiazoles, which antihypertensive activity was determined, were used [6, 8-9]. Calculation of molecular descriptors was carried out using Hyper-Chem 7.5 software [10] (license on HyperChem 7.5 software is available for Danylo Halytsky Lviv National Medical University); BuildQSAR software was used for QSAR-model building [11]. 


\section{Results}

Target polysubstituted functionalized aminothiazoles were synthesized as described $[6,8-9]$. The structures of the test compounds are presented in Fig. 1.

Antihypertensive activity (HA) studies were performed in vivo experiments on laboratory white rats [9]. The activity of the test compounds was determined by a decrease in the blood pressure in the tail artery of the animal in 60 minutes after intraperitoneal injection. For screening studies of antihypertensive activity, substances at a dose of $7 \mathrm{mg} / \mathrm{kg}$ were used. The antihypertensive activity of the test compounds are presented in Table 1.

The 2D structures of the molecules of the test compounds were converted into 3D using Hyper-Chem 7.5 software [10]. The optimization of the structures of the tested substances was carried out by the method of molecular mechanics $\mathrm{MM+}$, and the final energy minimization was carried out by semi-empirical quantumchemical AM1 method until a standard deviation of $0.001 \mathrm{kcal} / \mathrm{mol}$ was achieved. The calculation of number of descriptors (electronic, steric, geometric, energy) $[12,13]$ was carried out for the tested compounds. The molecular descriptors were calculated using Hyper-Chem 7.5: the surface and grid area of the molecule
(SA and SG), volume of the molecule (V), lipophilicity parameter $(\log P)$, refractivity $(R)$, polarizability $(P)$, molecular weight $(M)$, dipole moment (D). The parameters of the calculated molecular descriptors of the test compounds are presented in Table. 1.

The following parameters were also calculated for Oxygen (Ch_O), Sulfur (Ch_S), Nitrogen of thiazole cycle (Ch_Nt) and Nitrogen of amino-group (Ch_N); distances between atoms of Sulfur and Oxygen (D_S-O), Sulfur and Nitrogen Nt (D_S-Nt), Nitrogen of the aminogroup and Nitrogen of the thiazole cycle (D_Nt$\mathrm{N})$; angles between the atoms of the Nitrogen of the thiazole cycle, Sulfur and Nitrogen of the amino group (A_N-S-Nt), Nitrogen of the amino group, Oxygen and Nitrogen of the thiazole cycle (A_N-O-Nt). The attained parameters are presented in Table 2.

The energy parameters of the studied molecules were assessed: total energy (TE), binding energy (BE), isolated atomic energy (IAE), electronic energy $(E E)$, core-core interaction (CCI), heat of formation (HF), energies of the highest occupied molecular orbital and lowest unoccupied molecular orbital (HOMO and LUMO), hydration energy (EH). The attained results of energy parameters are presented in Table 3.<smiles>[R]c1ccc(/N=c2/sc([R])c(-c3ccccc3)n2[R])cc1</smiles>

1-3<smiles>[R]C(=O)c1s/c(=N\c2cccc([R])c2)n([R])c1C</smiles>

4-13

1. $\mathbf{R}=4-\mathrm{OMe}, \mathbf{R}^{\mathbf{1}}=2,5-$ diMe, $\mathbf{R}^{\mathbf{2}}=\left(\mathrm{CH}_{2}\right)_{2} \mathrm{OH}, \mathbf{R}^{\mathbf{3}}=\mathrm{H}$

2. $\mathbf{R}=4-\mathrm{OMe}, \mathbf{R}^{\mathbf{1}}=4-\mathrm{OCHF}_{2}, \mathbf{R}^{\mathbf{2}}=\left(\mathrm{CH}_{2}\right)_{3} \mathrm{OH}, \mathbf{R}^{3}=\mathrm{Me}$

3. $\mathbf{R}=4-\mathrm{OCHF}_{2}, \mathbf{R}^{\mathbf{1}}=4-\mathrm{OMe}, \mathbf{R}^{2}=\left(\mathrm{CH}_{2}\right)_{2} \mathrm{OH}, \mathbf{R}^{3}=\mathrm{H}$

4. $\mathbf{R}^{\mathbf{1}}=\mathrm{H}, \mathbf{R}^{\mathbf{2}}=\mathrm{N}$-piperydinyl $\left(\mathrm{CH}_{2}\right)_{2}, \mathbf{R}^{\mathbf{3}}=\mathrm{H}$,

5. $\mathbf{R}^{\mathbf{1}}=4$-OMe, $\mathbf{R}^{\mathbf{2}}=\mathrm{N}$-morpholinyl $\left(\mathrm{CH}_{2}\right)_{2}-, \mathbf{R}^{\mathbf{3}}=\mathrm{OEt}$,

6. $\mathbf{R}^{\mathbf{1}}=2,3-$ diMe, $\mathbf{R}^{2}=\left(\mathrm{CH}_{2}\right)_{2} \mathrm{OH}, \mathbf{R}^{\mathbf{3}}=\mathrm{OEt}$,

Fig. 1. Structures of the tested compounds.<smiles>COc1ccc(CCN=c2sc(C(C)=O)c(C)n2CCc2ccc(OC)c(OC)c2)cc1OC</smiles><smiles>Clc1ccc(-c2cs/c(=N/C3CCCCC3)n2C2CCCCC2)cc1</smiles>

7. $\mathbf{R}^{\mathbf{1}}=4-\mathrm{Me}, \mathbf{R}^{\mathbf{2}}=3$ - pyridinyl- $\mathrm{CH}_{2}, \mathbf{R}^{\mathbf{3}}=\mathrm{H}_{\text {, }}$

8. $\mathbf{R}^{\mathbf{1}}=4-\mathrm{Me}, \mathbf{R}^{\mathbf{2}}=\mathrm{N}$-morpholinyl, $\mathbf{R}^{3}=\mathrm{H}$,

9. $\mathbf{R}^{1}=4-\mathrm{OEt}, \mathbf{R}^{\mathbf{2}}=\mathrm{N}$-morpholinyl, $\mathbf{R}^{3}=\mathrm{H}$,

10. $\mathbf{R}^{1}=4-\mathrm{Me}, \mathbf{R}^{2}=3,4-$ diOMe- $\mathrm{C}_{6} \mathrm{H}_{3}\left(\mathrm{CH}_{2}\right)_{2}, \mathbf{R}^{3}=\mathrm{H}$,

11. $\mathbf{R}^{1}=\mathrm{H}, \mathbf{R}^{2}=3,4$-diOMe- $\mathrm{C}_{6} \mathrm{H}_{3}\left(\mathrm{CH}_{2}\right)_{2}, \mathbf{R}^{3}=\mathrm{H}$,

12. $\mathbf{R}^{\mathbf{1}}=2-\mathrm{Me}, \mathbf{R}^{\mathbf{2}}=3,4-\operatorname{diOMe}-\mathrm{C}_{6} \mathrm{H}_{3}\left(\mathrm{CH}_{2}\right)_{2}, \mathbf{R}^{3}=\mathrm{H}$,

13. $\mathbf{R}^{1}=2,3-$ diMe, $\mathbf{R}^{2}=3,4-\operatorname{diOMe}-\mathrm{C}_{6} \mathrm{H}_{3}\left(\mathrm{CH}_{2}\right)_{2}, \mathbf{R}^{3}=\mathrm{H}$, 
Table 1. Antihypertensive activity and molecular descriptors of polysubstituted functionalized aminothiazoles

\begin{tabular}{|c|c|c|c|c|c|c|c|c|c|}
\hline No. & AH & SA & SG & V & $\log P$ & $R$ & $P$ & $M$ & $D$ \\
\hline 1 & 8 & 546.8 & 667.17 & 1142.6 & 1.3 & 122.22 & 43.77 & 435.38 & 1.99 \\
\hline 2 & 11.9 & 705.14 & 731.37 & 1248.5 & 0.93 & 130.69 & 46.06 & 501.39 & 7.11 \\
\hline 3 & 10.5 & 642.6 & 666.28 & 1141.6 & 0.85 & 120.04 & 42.39 & 473.33 & 4.49 \\
\hline 4 & 6.2 & 551.29 & 605.91 & 1063.4 & 1.22 & 113.24 & 41.49 & 380.94 & 3.29 \\
\hline 5 & 14,3 & 666.39 & 680.82 & 1224.9 & 1.7 & 124.63 & 45.78 & 470.42 & 7.44 \\
\hline 6 & 5.5 & 514.36 & 560.73 & 969.72 & 0.68 & 99.07 & 36.3 & 334.43 & 4.21 \\
\hline 7 & 8.3 & 511.78 & 577 & 981.94 & 0.68 & 107.71 & 38.47 & 337.44 & 2.2 \\
\hline 8 & 6.6 & 570.15 & 579.33 & 1015.7 & 0.68 & 106.46 & 38.94 & 367.89 & 2.83 \\
\hline 9 & 8.2 & 618.67 & 628.25 & 1094.2 & -0.12 & 113.31 & 41.41 & 397.92 & 3.07 \\
\hline 10 & 7.8 & 588.77 & 639.32 & 1147.8 & 0.42 & 128.78 & 45.96 & 410.53 & 2.04 \\
\hline 11 & 12.2 & 549.76 & 614.72 & 1100.6 & 0.27 & 124.5 & 44.1 & 396.5 & 2.24 \\
\hline 12 & 2.1 & 567.3 & 629.3 & 1137.4 & 0.42 & 106.94 & 42.56 & 410 & 2.49 \\
\hline 13 & 11.1 & 659 & 672.16 & 1266 & 1.36 & 142.63 & 51.19 & 505.47 & 3.97 \\
\hline 14 & 9.2 & 692.98 & 743.14 & 1354 & -0.94 & 146.17 & 52.54 & 484.86 & 3.72 \\
\hline 15 & 10.8 & 530.62 & 639.61 & 1153.6 & 4.88 & 123.15 & 46.06 & 455.88 & 2.28 \\
\hline
\end{tabular}

Table 2. Charges, distances, angles between atoms of polysubstituted functionalized aminothiazoles

\begin{tabular}{|c|c|c|c|c|c|c|c|c|c|c|}
\hline No. & Ch_O & Ch_S & Ch_Nt & Ch_N & D_S-O & D_S-Nt & D_S-N & D_Nt-N & A_N-S-Nt & A_N-O-Nt \\
\hline 1 & 0 & 0.382 & -0.204 & -0.243 & 0 & 2.59 & 2.79 & 2.37 & 52.27 & 0 \\
\hline 2 & 0 & 0.285 & -0.227 & -0.205 & 0 & 2.60 & 2.79 & 2.38 & 52.41 & 0 \\
\hline 3 & 0 & 0.352 & -0.215 & -0.229 & 0 & 2.59 & 2.79 & 2.38 & 52.31 & 0 \\
\hline 4 & -0.281 & 0.392 & -0.204 & -0.24 & 3.53 & 2.59 & 2.79 & 2.37 & 52.32 & 21.86 \\
\hline 5 & -0.363 & 0.449 & -0.227 & -0.207 & 2.6 & 2.61 & 2.63 & 2.49 & 56.86 & 22.08 \\
\hline 6 & -0.354 & 0.489 & -0.243 & -0.214 & 3.37 & 2.60 & 2.63 & 2.50 & 56.93 & 23.3 \\
\hline 7 & -0.271 & 0.384 & -0.209 & -0.223 & 3.34 & 2.59 & 2.79 & 2.38 & 52.42 & 22.16 \\
\hline 8 & -0.268 & 0.366 & -0.117 & -0.191 & 3.42 & 2.63 & 2.77 & 2.41 & 51.79 & 32.96 \\
\hline 9 & -0.261 & 0.374 & -0.125 & -0.193 & 3.27 & 2.62 & 2.77 & 2.41 & 53.1 & 23.14 \\
\hline 10 & -0.287 & 0.405 & -0.203 & -0.248 & 3.16 & 2.59 & 2.78 & 2.38 & 52.5 & 23 \\
\hline 11 & -0.286 & 0.406 & -0.202 & -0.25 & 3.17 & 2.59 & 2.78 & 2.38 & 52.53 & 23.04 \\
\hline 12 & -0.285 & 0.405 & -0.203 & -0.251 & 3.18 & 2.59 & 2.78 & 2.38 & 52.4 & 22.85 \\
\hline 13 & -0.286 & 0.361 & -0.206 & -0.238 & 3.13 & 2.59 & 2.78 & 2.38 & 52.37 & 23.13 \\
\hline 14 & -0.282 & 0.356 & -0.211 & -0.273 & 3.23 & 2.6 & 2.76 & 2.4 & 53 & 22.94 \\
\hline 15 & 0 & 0.292 & -0.183 & -0.251 & 0 & 2.64 & 2.76 & 2.41 & 52.97 & 0 \\
\hline
\end{tabular}

In the study, the optimal set of molecular descriptors was chosen by a sequential algorithm and the Multiple Linear Regression (MLR) method to obtain QSAR models by means of BuildQSAR [11]. Statistically, the number of compounds tested $(\mathrm{N})$ and independent variables (M) used in the model should correspond to a ratio of $N / M \geq 5$. Descriptors with high pairwise correlation were excluded from the multidimensional descriptor space. Firstly, the descriptors from different groups were used to build individual models, and then descriptors that most fully describe the change in biological activity were used to obtain mixed models. This allowed us to choose single or multi-parameter models with the maximal correlation coefficient $(r)$ and the minimal standard deviation (s). The models were further investigated for their adequacy by means of the Fisher coefficient $(F)$ and predictive ability using the $\mathrm{Q}^{2}$ crossvalidation coefficient and sum of squares of prediction error (SRRESS).

Based on the calculated molecular descriptors and the parameter of antihypertensive activity, the QSAR models were derived $\mathbf{A H}=\mathbf{a}+$ $\mathbf{b} \cdot \mathbf{X} \mathbf{1}+\mathbf{c} \cdot \mathbf{X} \mathbf{2}+\mathbf{d} \cdot \mathbf{X} \mathbf{3}$, where the activity parameter $\mathrm{AH}$ is antihypertensive activity and X1, X2, X3 are molecular descriptors: 
Table 3. Energy parameters of polysubstituted functionalized aminothiazoles

\begin{tabular}{|c|c|c|c|c|c|c|c|c|c|}
\hline No. & TE & BE & IAE & EE & CCI & HF & HOMO & LUMO & EH \\
\hline 1 & -103278 & -5050 & -98228 & -780638 & 677360 & 4.33 & -7.81 & -0.27 & -5.71 \\
\hline 2 & -136024 & -5476 & -130548 & -989792 & 853768 & -153.1 & -8.12 & -0.56 & -12.2 \\
\hline 3 & -128838 & -4913.8 & -123924 & -872337 & 743499 & -141.4 & -7.92 & -0.59 & -11.9 \\
\hline 4 & -100651 & -4965.6 & -95685 & -785689 & 685038 & 19.91 & -7.97 & -0.34 & -3.82 \\
\hline 5 & -117058 & -5558.6 & -111499 & -960851 & 843794 & -71.13 & -8.34 & -0.48 & -2.35 \\
\hline 6 & -93372 & -4600.7 & -88771 & -679708 & 586336 & -78.24 & -8.26 & -0.42 & -5.84 \\
\hline 7 & -88276 & -4631.9 & -83644 & -648490 & 560214 & 69.86 & -8.01 & -0.41 & -3.63 \\
\hline 8 & -99334 & -4601.6 & -94733 & -733476 & 634141 & 3.26 & -8.22 & -0.45 & -2.42 \\
\hline 9 & -110306 & -4971.6 & -105334 & -827964 & 717658 & -32.06 & -8.05 & -0.45 & -4.36 \\
\hline 10 & -112313 & -5773 & -106539 & -940854 & 828541 & -17.24 & -7.93 & -0.48 & -3.82 \\
\hline 11 & -108718 & -5490 & -103228 & -889289 & 780571 & -9.39 & -8.03 & -0.489 & -5.25 \\
\hline 12 & -112312 & -5772 & -106539 & -952370 & 840058 & -16.28 & -7.95 & -0.46 & -4.33 \\
\hline 13 & -124384 & -6144 & -118239 & -1097703 & 973319 & -34.41 & -8.12 & -0.65 & -3.25 \\
\hline 14 & -137848 & -6793 & -131054 & -1237984 & 1100137 & -92.91 & -8.19 & -0.21 & -6.53 \\
\hline 15 & -101712 & -5379 & -96332 & -842684 & 740972 & 15.79 & -8.21 & -0.31 & 0.58 \\
\hline
\end{tabular}

$\mathbf{H A}=-0.044( \pm 0.019) \mathbf{S A}+0.041( \pm 0.011) \mathbf{V}$ -24.062( \pm 7.933$)$ LUMO-21.062 $( \pm 8.297)(1)$

$\left(n=015 ; r=0.936 ; s=1.171 ; F=26.006 ; Q^{2}=0.799 ;\right.$ SPRESS $=1.492)$

HA $=+0.048( \pm 0.015) \mathbf{V}+0.004( \pm 0.001)$ IAE

-27.025( \pm 9.356$)$ LUMO-29.957( \pm 10.172$)(2)$

$\left(n=015 ; r=0.930 ; s=1.227 ; F=23.353 ; Q^{2}=0.718 ;\right.$ SPRESS $=1.767)$

$\mathbf{H A}=+0.019( \pm 0.011) \mathbf{V}+0.731( \pm 0.866) \log \mathbf{P}-$

13.142( \pm 9.671$)$ LUMO-19.284( \pm 13.242$)$ (3)

$\left(n=015 ; r=0.825 ; s=1.879 ; F=7.844 ; Q^{2}=0.500 ;\right.$ SPRESS $=2.354)$

$\mathbf{H A}=+0.051( \pm 0.028) \mathbf{R}-0.002( \pm 0.001) \mathbf{B E}-$

17.152( \pm 10.844$)$ LUMO -16.857( \pm 13.668$)$ (4)

$\left(n=015 ; r=0.813 ; s=1.940 ; F=7.132 ; Q^{2}=0.086 ;\right.$ SPRESS $=3.470)$

$\mathbf{H A}=+0.033( \pm 0.026) \mathbf{R}+0.423( \pm 0.304)$

P-17.522( \pm 10.543$)$ LUMO $-20.150( \pm 14.952)$ (5)

$\left(n=015 ; r=0.825 ; s=1.880 ; F=7.840 ; Q^{2}=0.295 ;\right.$ SPRESS $=2.795)$

$\mathbf{H A}=+1.237( \pm 0.685) \mathbf{P}+0.002( \pm 0.001) \mathbf{E} \mathbf{E}-$

$22.339( \pm 12.913)$ LUMO-36.725 $( \pm 21.841)$ (6)

$\left(n=015 ; r=0.817 ; s=1.920 ; F=7.359 ; Q^{2}=0.313 ;\right.$ SPRESS $=2.760)$

$\mathbf{H A}=+1.165( \pm 0.669) \mathbf{P}-0.002( \pm 0.001) \mathbf{C C I}-$ 20.886( \pm 12.661$)$ LUMO-35.268( \pm 21.945$)$ (7)

$\left(n=015 ; r=0.807 ; s=1.968 ; F=6.835 ; Q^{2}=0.296 ;\right.$ SPRESS $=2.793)$

$\mathbf{H A}=+0.019( \pm 0.012) \mathbf{V}-11.777( \pm 10.599)$ LUMO+0.275( \pm 0.771$)$ A_N-S-Nt-32.425( \pm 42.963$)$ (8)

$\left(n=015 ; r=0.778 ; s=2.092 ; F=5.624 ; Q^{2}=0.115 ;\right.$ SPRESS $=3.132)$

$\mathbf{H A}=+0.019( \pm 0.012) \mathbf{V}-11.197( \pm 11.685)$ LUMO+6.871 ( \pm 57.283$)$ Ch_N-16.433( \pm 20.007$)$ (9)

$\left(n=015 ; r=0.766 ; s=2.142 ; F=5.196 ; Q^{2}=0.158\right.$; SPRESS $=3.055$ )
$\mathbf{H A}=+0.526( \pm 0.373) \mathbf{P}-12.807( \pm 11.969)$

L UMO+19.091( \pm 33.934$)$ D_N - Nt - 65.167 $( \pm 86.579)(10)$

$\left(n=015 ; r=0.721 ; s=2.307 ; F=3.973 ; Q^{2}=0.028 ;\right.$ SPRESS $=3.284)$

$\mathbf{H A}=+0.023( \pm 0.012) \mathbf{V}+0.233( \pm 0.391) \mathbf{E H}-$ 14.248( \pm 10.989$)$ LUMO-21.927( \pm 15.461$)$ (11)

$\left(n=015 ; r=0.799 ; s=2.001 ; F=6.492 ; Q^{2}=0.351\right.$; SPRESS $=2.683$ )

$\mathbf{H A}=+0.012( \pm 0.015) \mathbf{V}+0.535( \pm 1.072)$ $\log \mathbf{P}+0.528( \pm 0.934) \mathbf{D}-6.815( \pm 14.912)(12)$

$\left(n=015 ; r=0.705 ; s=2.362 ; F=3.622 ; Q^{2}=0.048 ;\right.$ SPRESS $=3.409$ )

\section{Discussion}

According to the analysis of the derived QSAR models (1-12), it was established that the antihypertensive activity increases with the increase of the parameters of the following molecular descriptors: volume, lipophilicity, dipole moment, refractivity and polarization of the molecule and decrease of the surface area of the molecule. Among the energy parameters, the most significant effect is the energy of the lowest unoccupied molecular orbital, with increasing antihypertensive activity and decrease in this parameter. Antihypertensive activity also increases with increasing charge on the Nitrogen atom of the amino group, with increasing distance between this atom and the Nitrogen atom of the thiazole cycle and increase of the angle A_N-S-Nt.

The dependence of the observed and predicted antihypertensive activities for the QSAR models (1-3) is presented in Fig. 2.

The search for quantitative regularities of the dependence of the antihypertensive activity 

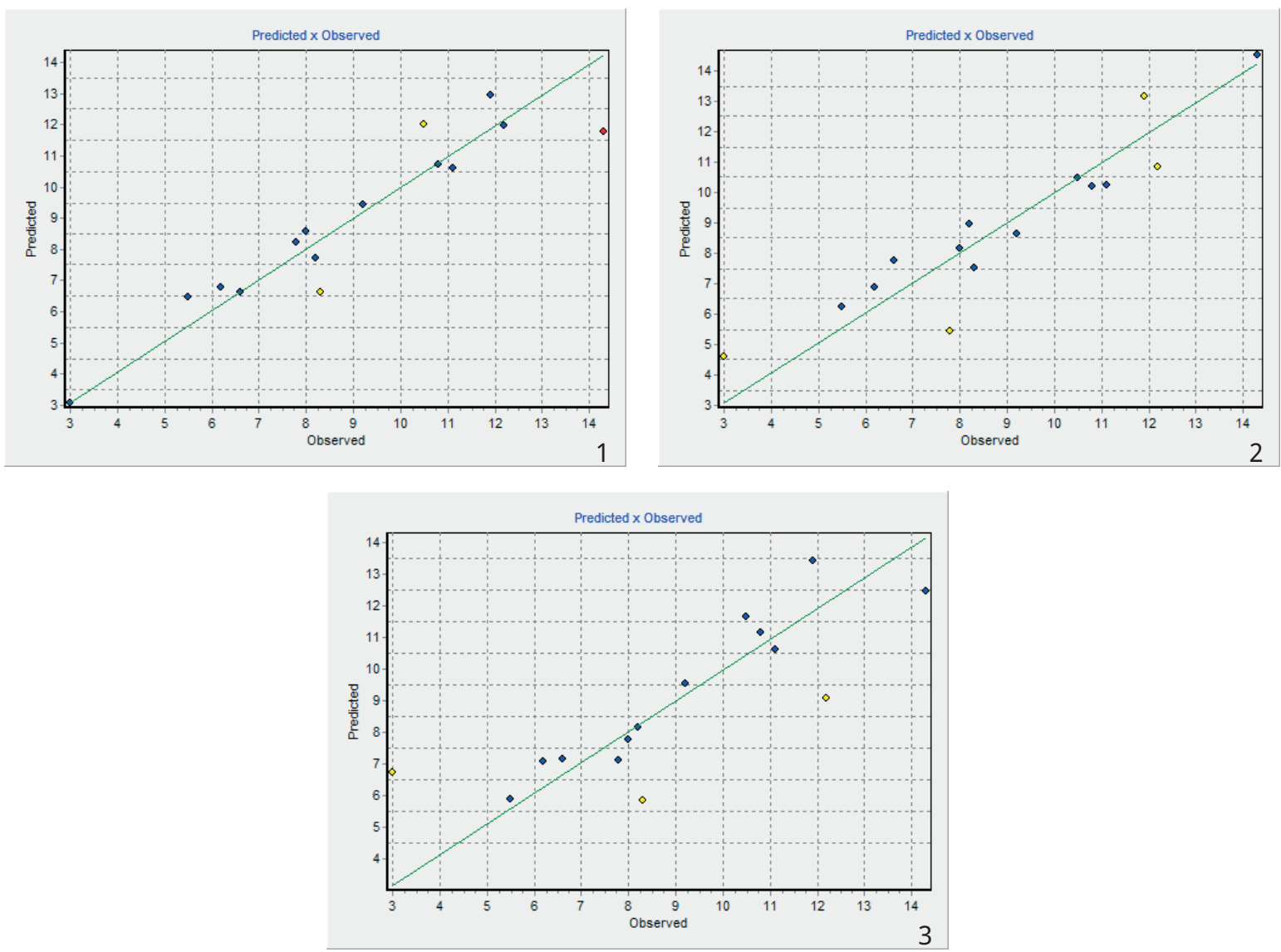

Fig. 2. The dependence of the observed and predicted antihypertensive activities for the QSAR models $1,2,3$.

of the studied compounds on the parameters of their molecular descriptors allowed deriving statistically qualitative QSAR models (1-3) $(r=0,825 \div 0,936)$ characterized by sufficient adequacy $(\mathrm{F}=7.8 \div 26.006)$ and predictive ability $\left(\mathrm{Q}^{2}=0.5-0.799\right)$.

\section{Conclusions}

Thus, according to the study of the 'structure-antihypertensive' activity relationship for polysubstituted functionalized aminothiazoles, the QSAR analysis revealed that the volume, area, lipophilicity, dipole moment, refractivity, polarization of the molecule and energy of the lowest unoccupied molecular orbital have the most significant effect on antihypertensive activity. The derived QSAR-models are suggested for antihypertensive activity prediction within abovementioned row of compounds and can be a theoretical basis for de novo development of new potential antihypertensive drugs.

\section{Funding}

This research received no external funding. Conflict of Interests

The authors declare no conflict of interest.

\section{QSAR-АНАЛІЗ ПОЛІЗАМІЩЕНИХ ФУНКЦІОНАЛІЗОВАНИХ АМІНОТІАЗОЛІВ 3 ГІПОТЕНЗИВНОЮ АКТИВНІСТЮ}

I.B. Драпак ЛЬВІВСЬКИЙ НАЦІОНАЛЬНИЙ МЕДИЧНИЙ УНІВЕРСИТЕТ ІМЕНІ ДАНИЛА ГАЛИЦЬКОГО, УКРАЇНА 
Мета дослідження. Визначення молекулярних дескрипторів для QSAR аналізу полізаміщених функціоналізованих амінотіазолів як теоретичної основи для цілеспрямованого пошуку нових антигіпертензивних засобів.

Методи дослідження. Розрахунок молекулярних дескрипторів ma побудова QSAR-моделей проводились з використанням програмних пакетів: Hyper-Chem 7.5 ma BuildQSAR.

Результати. Для 15 нових полізаміщених функціоналізованих амінотіазолів із встановленою in vivo гіпотензивною активністю проведено розрахунок ряду молекулярних дескрипторів (електронних, стеричних, геометричних, енергетичних). На основі розрахованих молекулярних дескрипторів та параметра гіпотензивної активності були отримані QSAR-моделі: $A H=a+b \cdot X 1+c \cdot X 2+d \cdot X 3$, де AH - гіпотензивна активність, а X1, Х2, Х3 - молекулярні дескриптори.

Висновки. Проведено дослідження зв'язку "структура - гіпотензивна активність" для ряду полізаміщених функціоналізованих амінотіазолів. За результатами QSAR-aналізу встановлено, що об'єм, площа, ліпофільність, дипольний момент, рефрактивність, поляризованість молекули та енергія нижчої незайнятої молекулярної орбіталі мають найбільш значний вплив на гіпотензивну активність. Одержані QSAR-моделі будуть використані для прогнозування активності даного ряду сполук і можуть розглядатися як теоретична основа для de поvо дизайну нових потенційних гіпотензивних препаратів.

КЛЮЧОВІ СЛОВА: полізаміщені функціоналізовані амінотіазоли; гіпотензивна активність; молекулярні дескриптори; QSAR-аналіз.

\section{Інформація про автора}

Драпак I.в. - кандидат фармацевтичних наук, доцент кафедри загальної, біонеорганічної та фізколоїдної хімії Львівського національного медичного університету імені Данила Галицького

\section{Information about the author}

Drapak I.V. - PhD, Associate Professor of the Department of General, Bioinorganic, Physical and Colloidal Chemistry, Danylo Halytsky Lviv National Medical University, Lviv, Ukraine.

ORCID 0000-0002-6830-2908, e-mail: iradrapak@ukr.net

\section{References}

1. Cherkasov A, Muratov EN, Fourches D, Varnek A, Baskin II, Cronin M, Dearden J, Gramatica P, Martin YC, Todeschini R, Consonni V. QSAR modeling: where have you been? Where are you going to? Journal of Medicinal Chemistry. 2014 Jan 6;57(12): 4977-5010.

doi:10.1021/jm4004285

2. Wang T, Wu MB, Lin JP, Yang LR. Quantitative structure - activity relationship: promising advances in drug discovery platforms. Expert Opinion on drug Discovery. 2015 Dec 2;10(12):1283-300.

doi: 10.1517/17460441.2015.1083006

3. Tetko IV, Gasteiger J, Todeschini R, Mauri A Livingstone D, Ertl P, Palyulin VA, Radchenko EV, Zefirov NS, Makarenko AS, Tanchuk VY. Virtual computational chemistry laboratory-design and description. Journal of Computer-Aided Molecular Design. 2005 Jun 1;19(6):453-63.

doi: 10.1007/s10822-005-8694-y

4. Giri RS, Thaker HM, Giordano T, Williams J, Rogers D, Sudersanam V, Vasu KK. Design, synthesis and characterization of novel 2-(2, 4-disubstitutedthiazole-5-yl)-3-aryl-3H-quinazoline-4-one derivatives as inhibitors of NF-KB and AP-1 mediated transcription activation and as potential anti-inflammatory agents. European journal of medicinal chemistry. 2009 May 1;44(5):2184-9.

doi: 10.1016/j.ejmech.2008.10.031

5. Abdel-Wahab BF, Mohamed SF, Amr AE, Abdalla MM. Synthesis and reactions of thiosemicarbazides, triazoles, and Schiff bases as antihyper- tensive a-blocking agents. Monatshefte für ChemieChemical Monthly. 2008 Sep 1;139(9):1083-90.

doi: 10.1007/s00706-008-0896-2

6. Drapak I, Perekhoda L, Demchenko N, Suleiman M, Rakhimova M, Demchuk I, Taran S, Seredynska N, Gerashchenko I. Cardioprotective Activity of Some 2-Arylimino-1, 3-Thiazole Derivatives. Scientia Pharmaceutica. 2019 Mar;87(1):7-8. doi: $10.3390 /$ scipharm87010007

7. Perekhoda L, Yeromina H, Drapak I, Kobzar N, Smolskiy O, Demchenko N. The antioxidant properties of 1-[2-(R-phenylimino)-4-methyl-3-(3-[morpholine4-yl] propyl)-2, 3-dihydro-1, 3-thiazol-5-yl] ethane-1one derivatives under conditions of artificial oxidative stress in vitro. Saudi Journal of Medical and Pharmaceutical Sciences. 2017;3(1):55-9.

doi: $10.21276 /$ sjmps.2017.3.9

8. Yeromina HO, Drapak IV, Perekhoda LO, Yaremenko VD, Demchenko AM, Perekhoda LA. Synthesis of 2-(4-aryl (adamantyl)-2-phenyliminothiazol-3-yl)-ethanol derivatives and prediction of their biological activity. Der Pharma Chemica. 2016;8(3):64-70.

9. Drapak IV, Zimenkovsky BS, Seredynska NM, Demchenko AM. 1-\{3-[2-(3,4-Dimethoxy-phenyl)ethyl]-4-methyl-1-2-phenylimino-2,3-dihydro-thiazol5-yl\}-ethanone hydrochloride, which is antihypertensive action. No 19960 UA Patent UA. 2019 August 27.

10. Laxmi K. Structural Elucidation of Drug Aspirin by Using Various Software Tools Like Hyper- 
Chem, Argus Lab, ChemSketch, Avogrado and Chemeo Database. J Theor Comput Sci. 2019;5(163):2.

11. De Oliveira DB, Gaudio AC. BuildQSAR: a new computer program for QSAR analysis. Quantitative Structure-Activity Relationships: An International Journal Devoted to Fundamental and Practical Aspects of Electroanalysis. 2000 Dec;19(6):599-601. doi: 10.1002/1521-3838(200012)19:6\%3C599:: AID-QSAR599\%3E3.0.CO;2-B
12. Todeschini R, Consonni V. Handbook of Molecular Descriptors. 2000. NY, Toronto: Wiley-VCH. doi: $10.1002 / 9783527613106$

13. Todeschini R, Consonni V. Molecular descriptors for chemoinformatics: volume I: alphabetical listing/volume II: appendices, references. John Wiley \& Sons; 2009 Oct 30.

doi: $10.1002 / 9783527628766$

Received 05 September 2019; revised 29 October 2019; accepted 26 November 2019.

This is open access article distributed under the Creative Commons Attribution License, which permits unrestricted use, distribution, and reproduction in any medium, provided the original work is properly cited. 